\title{
46: Principles of Radiative Transfer
}

\author{
MATTHIAS DRUSCH ${ }^{1}$ AND SUSANNE CREWELL ${ }^{2}$ \\ ${ }^{1}$ European Centre for Medium-Range Weather Forecasts, Reading, UK \\ ${ }^{2}$ Munich University, Munich, Germany
}

\begin{abstract}
The article gives an introduction to classical vector radiative transfer theory (RTT). It comprises a brief summary of the fundamental quantities in RTT and the corresponding definitions. Based on these quantities, the transfer equation for radiation will be introduced in its basic form for a plane-parallel, horizontally homogeneous atmosphere. Polarization and the Stokes vector are introduced, which extend the scalar radiative transfer equation to the more general form of the vector radiative transfer equation. Scattering processes and interactions with the surface are discussed within the mathematical framework given in the first part of the paper. As an example, the theoretical concept presented is then applied to passive microwave remote sensing. A solution for the radiative transfer equation, which is commonly used for the retrieval of atmospheric quantities (e.g. water vapor) and land surface properties (e.g. soil moisture), is derived based on approximations and simplifications. More general approaches to solve the radiative transfer equation including multiple scattering are described and discussed in a separate section.
\end{abstract}

\section{INTRODUCTION}

Radiative transfer theory (RTT) describes the interaction between matter and electromagnetic radiation. Electromagnetic waves travel in vacuum and air at the speed of light at $\sim 3 \times 108 \mathrm{~ms}^{-1}$. The electromagnetic spectrum covers gamma rays, $\mathrm{X}$ rays, ultraviolet, visible, infrared, microwaves, television signals, and radio waves as shown in Figure 1. Following Planck's law, solar radiation can be described as radiation emitted from a black body at a temperature of about $6000 \mathrm{~K}$. Consequently, the solar component comprises radiation from the Gamma rays to the infrared spectral range. Only $\sim 0.4 \%$ of the energy of solar radiation is emitted at wavelengths above $5 \mu \mathrm{m}$. The irradiance at the top of the atmosphere (solar constant $S_{0}$ ) is $1366 \mathrm{Wm}^{-2} \pm 3 \mathrm{Wm}^{-2}$ depending on the solar-earth distance and natural fluctuations of the sun's activity (Lean and Rind, 1998). Consequently, the energy available for the earth-atmosphere system is:

$$
E_{\mathrm{sol}, \downarrow}=\pi r_{E}^{2}(1-\alpha) S_{0}
$$

with the planetary albedo $\alpha$. This energy is absorbed by the system and reemitted following Stefan-Boltzmann's law:

$$
E_{\mathrm{ter}, \uparrow}=4 \pi r_{E}^{2} \sigma_{B} T_{E}^{4}
$$

with the blackbody temperature of the earth $T_{E}$, the radius of the Earth $r_{E}$ and the Stefan-Boltzmann constant $\sigma_{B}$. Using equations (1) and (2), a blackbody temperature for the atmosphere-earth system of $\sim 255 \mathrm{~K}$ is obtained. At this temperature, a blackbody emits only $0.4 \%$ of its radiation at wavelengths below $5 \mu \mathrm{m}$ (Goody and Yung, 1961). As a consequence, solar and terrestrial radiation can be treated independently. In equations (1) and (2) radiation has been integrated over all wavelengths and angles. In addition, $T_{E}$ and $\alpha$ are "mean" quantities, which comprise the atmosphere (clouds, gaseous profiles, etc.), ocean (ice, foam, algae, etc.), and land surfaces (vegetation, soil texture, snow, etc.). For a comprehensive description of the interaction of electromagnetic waves and the earth-atmosphere system in hydrological applications and numerical modelling (including climate models, numerical weather prediction (NWP) models, soil-vegetation-atmosphere transfer 


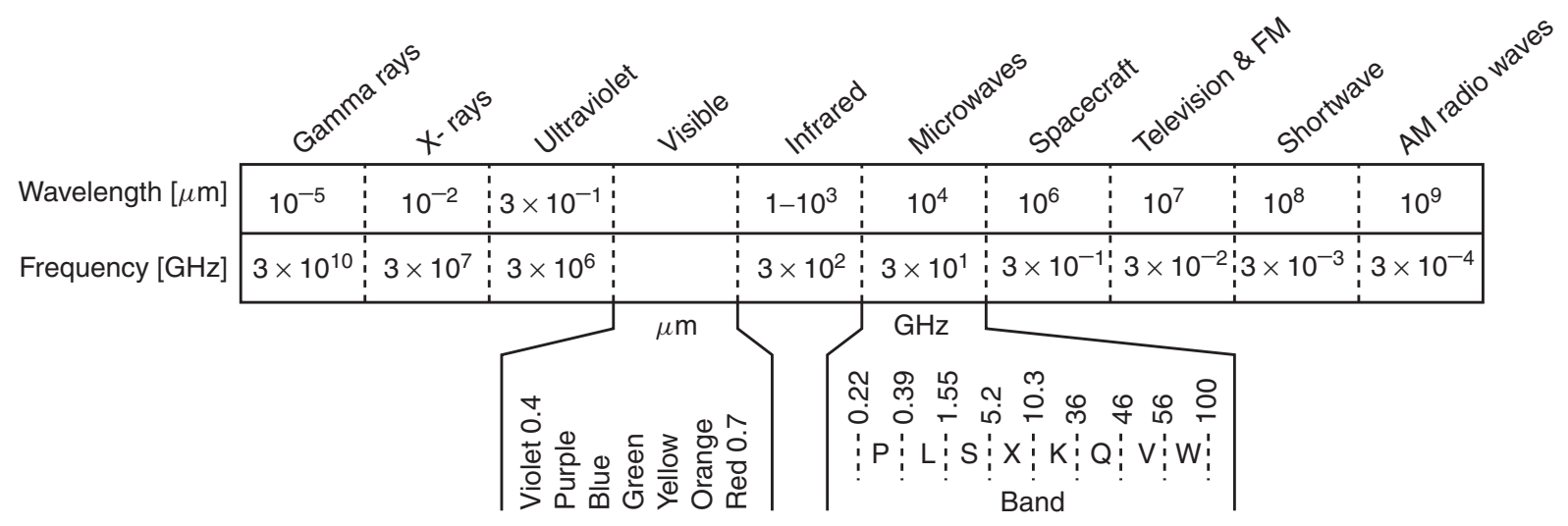

Figure 1 The electromagnetic spectrum (after Liou, 2002; Ulaby et al., 1982)

(SVAT) models, physical and chemical cloud models, vegetation growth models, etc.), the RTT has to be much more complex.

Remote sensing is a second field of research, which is strongly related to RTT. Passive remote sensing techniques make use of the interaction of natural radiation with the variable of interest while active methods employ artificial sources of radiation such as lasers or radio wave sources. Retrieving geophysical parameters from radiation measurements requires RTT unless purely statistical methods are used.

In general, the theoretical and computational work on RTT can be divided in forward (or direct) and backward (or inverse) applications. In forward modelling studies, the geophysical parameters characterizing the earth-atmosphere system and the boundary conditions for the radiation sources are prescribed and the radiation field is computed for the given environment. Numerical climate or NWP modelling are typical examples of forward applications. For the inverse problem, the radiation field is known and it is aimed to retrieve the parameters characterizing the atmosphere and/or the surface. Many of the remote sensing applications have to address the inverse problem. Since analytical solutions for the inverse problem do not exist, a large number of statistical and physical retrieval methods have been developed. Often, variational methods assume a certain set of geophysical parameters and the computed radiances are compared with the observations. In the consecutive iteration steps, the parameters are modified to match the observations taking into account the observation and model errors.

Even if we limit ourselves to forward applications, it is impossible to provide a complete introduction to RTT for the most relevant physical processes at the visible to microwave spectral range within the framework of an article. For the basic laws in RTT, for example, Planck's law, Wien's law, Stefan-Boltzmann law and Kirchhoff's law, the reader is referred to more general textbooks, for example, Salby (1996). The focus of this paper is the classical vector RTT, since it has found extensive applications in research and operations. An overview of radiative transfer theory requires the use of mathematical expressions. The mathematics presented in this article partly exceeds the formalism presented in more general textbooks, for example, Salby (1996). Compared to specific books on radiative transfer (e.g. Chandrasekhar (1960), Liou (2002)) the number of equations is small and the corresponding level of complexity is low. However, the basic equations and theory presented in this article allow the reader to cope with more specific scientific articles addressing radiative transfer, remote sensing, engineering, and biophysical applications. A further reduction of the number of equations would not lead to a better understanding of the topic.

The first two sections comprise a brief summary of the fundamental quantities in RTT and the corresponding definitions. Based on these quantities, the transfer equation for radiation will be introduced in its basic form for a plane parallel, horizontally homogeneous atmosphere. This derivation can be found in many articles and textbooks on remote sensing and radiative transfer (e.g. Ulaby et al., 1982; Liou, 2002; Chandrasekhar, 1960). In the section "Polarization and vector radiative transfer equation", polarization and the Stokes vector will be introduced, which extends the scalar radiative transfer equation to the general form of the vector radiative transfer equation. The sections "Scattering and phase functions" and "Boundary conditions - the surface" address scattering processes and interactions with the surface, respectively. Based on the theoretical framework presented in the first six sections, the radiative transfer equation is simplified and solved for passive microwave applications. More general approaches to solve the radiative transfer equation are presented in the section "Solutions". 


\section{DEFINITIONS}

In a medium that interacts with radiation at a specific frequency $v$, the specific intensity $I_{v}$ can vary from point to point and with the direction of propagation at each point. Following Chandrasekhar (1960), the specific (or monochromatic) intensity can be defined as:

$$
I_{v} \equiv I_{v}(x, y, z ; l, m, n ; t)
$$

where $(x, y, z)$ and $(l, m, n)$ define the point and the direction cosines and $t$ indicates the temporal dependence of intensity. The specific intensity is given in units of $\mathrm{Wm}^{-2} \mathrm{sr}^{-1} \mathrm{~Hz}^{-1}$ and is often referred to just as intensity (or radiance) for simplicity. The integration over the entire hemisphere gives the flux density $F_{v}$ also called irradiance, which is used in energetical studies. Integration over an infinitesimal frequency range $\mathrm{d} v$, the directions defining an element of the solid angle $\mathrm{d} \omega\left(\mathrm{d} \omega=\mathrm{d} \sigma / r^{2}=\sin \theta \mathrm{d} \theta \mathrm{d} \phi\right)$ and a time interval $\mathrm{d} t$, leads to the amount of energy $d E_{v}$ transported through the cross-section $\mathrm{d} \sigma$ (Figure 2):

$$
\mathrm{d} E_{v}=I_{v} \cos \theta \mathrm{d} \nu \mathrm{d} \sigma \mathrm{d} \omega \mathrm{d} t
$$

If the intensity $I_{v}$ at a given point is independent of direction, the radiation field is called isotropic. In this case, the relation $F_{v}=\pi I_{v}$ holds. Such a radiation field, which is characterized by constant intensities at each point, is said to be homogeneous. For practical applications in hydrology and atmospheric sciences, the medium (e.g. atmosphere, vegetation canopy, or the soil) that interacts with the radiation is often characterized by stratified plane-parallel layers with invariant physical properties. In a spherical coordinate system where $z$ is the height and $\theta$ and $\phi$ the polar and azimuthal angles, the corresponding specific intensity can be written as:

$$
I_{v} \equiv I_{\nu}(z, \theta, \phi ; t)
$$

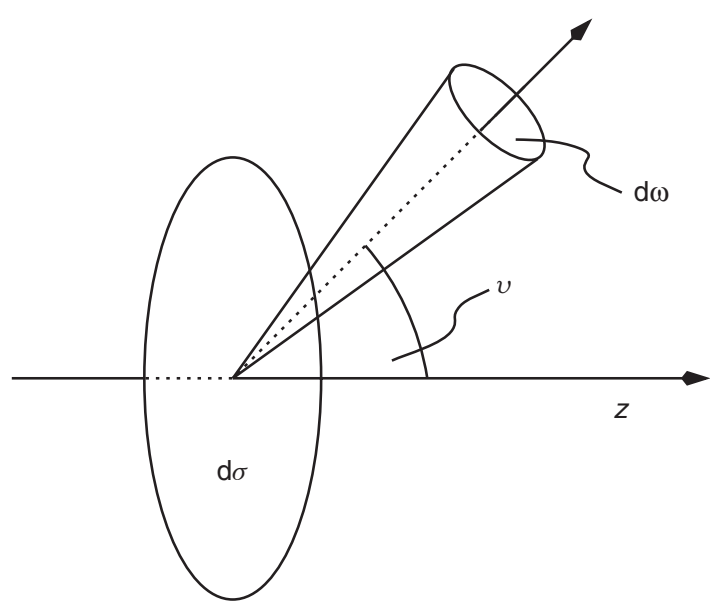

Figure 2 Definition of the specific intensity

\section{SCALAR RADIATIVE TRANSFER EQUATION}

Radiation characterized by a specific intensity $I_{v}$ passing a volume, defined by its thickness $\mathrm{d} s$ and a unit crosssection, will be weakened by an amount $\mathrm{d} I_{v}$. This extinction is caused by interaction with matter, namely absorption and/or scattering. Introducing the mass extinction, absorption, and scattering coefficients $\sigma_{e}^{\prime}, \sigma_{a}^{\prime}$, and $\sigma_{s}^{\prime}$ we can write:

$$
\sigma_{e}^{\prime}=\sigma_{a}^{\prime}+\sigma_{s}^{\prime}
$$

The corresponding volume coefficients $\sigma_{e}, \sigma_{a}$, and $\sigma_{s}$ can be obtained by multiplication with the density of the medium. Consequently, the amount of radiation absorbed and/or scattered by the matter in a volume $\mathrm{d} \sigma \mathrm{d} s$ for a solid angle element $\mathrm{d} \omega$ in a given time interval $\mathrm{d} t$ can be formulated as:

$$
\sigma_{e} \mathrm{~d} s I_{v} \mathrm{~d} v \mathrm{~d} \sigma \mathrm{d} \omega \mathrm{d} t
$$

On the other hand, radiation intensity can be increased within the volume by contributions from scattering and emission. This source term can be described by introducing the volume emission coefficient $j_{v}$

$$
j_{\nu} \mathrm{d} s \mathrm{~d} v \mathrm{~d} \sigma \mathrm{d} \omega \mathrm{d} t
$$

The change of the radiation intensity propagating along a path $\mathrm{d} s$ caused by extinction (7) and sources (8) expressed in specific intensity is therefore:

$$
\begin{aligned}
& \frac{\mathrm{d} I_{v}}{\mathrm{~d} s} \mathrm{~d} s \mathrm{~d} \nu \mathrm{d} \sigma \mathrm{d} \omega \mathrm{d} t=-\sigma_{e} \mathrm{~d} s I_{v} \mathrm{~d} \nu \mathrm{d} \sigma \mathrm{d} \omega \mathrm{d} t \\
& \quad+j_{v} \mathrm{~d} s \mathrm{~d} v \mathrm{~d} \sigma \mathrm{d} \omega \mathrm{d} t \text { or } \\
& \frac{\mathrm{d} I_{v}}{\sigma_{e} \mathrm{~d} s}=-I_{v}+\frac{j_{v}}{\sigma_{e}}
\end{aligned}
$$

The emission coefficient $j_{v}$ can be written as the sum of two source terms. Radiation in the direction defined through the angles $\theta$ and $\phi$ is due to scattering processes $j_{v s}$ and emission from the medium in local thermodynamic equilibrium, $j_{v a}$. The first term may be expressed as:

$$
\begin{aligned}
j_{v s}(\theta, \phi)= & \sigma_{s} \frac{1}{4 \pi} \int_{0}^{\pi} \int_{0}^{2 \pi} p\left(\theta, \phi ; \theta^{\prime}, \phi^{\prime}\right) I_{\nu}\left(\theta^{\prime}, \phi^{\prime}\right) \\
& \sin \theta^{\prime} \mathrm{d} \theta^{\prime} \mathrm{d} \phi^{\prime}
\end{aligned}
$$

In equation (10) the normalized phase function $p(\theta, \phi$, $\left.\theta^{\prime}, \phi^{\prime}\right)$ is introduced. It describes how much radiation is scattered from incidence direction characterized by angles $\left(\theta^{\prime}, \phi^{\prime}\right)$ into observation direction given by $(\theta, \phi)$. Perfect scattering in the absence of any absorption requires:

$$
\frac{1}{4 \pi} \int_{0}^{\pi} \int_{0}^{2 \pi} p\left(\theta, \phi ; \theta^{\prime}, \phi^{\prime}\right) \mathrm{d} \theta^{\prime} \mathrm{d} \phi^{\prime}=1
$$


Under the assumption of local thermodynamic equilibrium the thermal emission is given through Kirchhoff's law and the Planck function:

$$
\begin{aligned}
j_{v a} & =\sigma_{a} B_{v}(T) \\
B_{v}(T) & =\frac{2 h v^{3}}{c^{2}} \frac{1}{e^{h v / k_{B} T}-1}
\end{aligned}
$$

where $h$ and $k_{B}$ are the Planck and Boltzmann constants and $T$ the temperature. Combining equations (10) and (12) in (9) gives the scalar radiative transfer equation:

$$
\begin{aligned}
\frac{\mathrm{d} I_{v}}{\sigma_{e} \mathrm{~d} s}= & -I_{\nu}+\omega_{0} \frac{1}{4 \pi} \int_{0}^{\pi} \int_{0}^{2 \pi} p\left(\theta, \phi ; \theta^{\prime}, \phi^{\prime}\right) I_{\nu}\left(\theta^{\prime}, \phi^{\prime}\right) \\
& \sin \theta^{\prime} \mathrm{d} \theta^{\prime} \mathrm{d} \phi^{\prime}+\left(1-\omega_{0}\right) B_{v}(T)
\end{aligned}
$$

with $\omega_{0}$ the single scattering albedo defined as:

$$
\omega_{0}=\frac{\sigma_{s}}{\sigma_{s}+\sigma_{a}}
$$

It has to be noted that equation (14) is valid for a specific frequency only. Therefore, the properties of the medium may be frequency dependent as well. RTT in which the radiation directly depends on frequency and where conversion of radiation between different frequencies takes place will not be discussed. Active remote sensing techniques such as, Raman lidar may need other forms of the radiative transfer equation. Methods to solve equation (14) are discussed in the section "Solutions".

\section{POLARIZATION AND VECTOR RADIATIVE TRANSFER EQUATION}

Equation (14) describes the radiative transfer for the specific intensity $I_{v}$. Another source of information in remote sensing is the polarization of radiation. In order to model polarization effects due to particle scattering and/or rough surface reflection, we have to extend equation (14) to the full vector equation.

\section{Wave Formalism}

Elliptically polarized electromagnetic waves are described by the time-dependent electric field $\boldsymbol{E}$. Following Chandrasekhar (1960), Liou (1992) the field components can be projected to two perpendicular vectors in the plane orthogonal to the direction of propagation:

$$
\boldsymbol{E}(t)=\boldsymbol{E}_{l}(t)+\boldsymbol{E}_{r}(t)=a_{l}(t) \cdot \boldsymbol{e}_{l}+a_{r}(t) \cdot \boldsymbol{e}_{r}
$$

$\boldsymbol{e}_{l}$ and $\boldsymbol{e}_{r}$ are orthogonal unit vectors parallel and perpendicular to the scattering/reflection plane and define the local coordinate system as shown in Figure 3. In this system, the amplitudes and the complex electric field components can be expressed using the circular frequency $\omega_{c}$, the wave number $k$, four constants $\left(a_{l}, a_{r}, \varepsilon_{l}, \varepsilon_{r}\right)$, and the phase lag $\rho=\varepsilon_{l}-\varepsilon_{r}$ between both components of the wave:

$$
\begin{aligned}
& a_{l}=a_{l}^{0} \sin \left(\omega_{c} t-\varepsilon_{l}\right) \\
& a_{r}=a_{r}^{0} \sin \left(\omega_{c} t-\varepsilon_{r}\right) \\
& E_{l}=a_{l}^{0} e^{-i \rho} e^{-k z+i \omega_{c} t} \\
& E_{r}=a_{r}^{0} e^{-i \rho} e^{-k z+i \omega_{c} t}
\end{aligned}
$$

\section{Intensity Formalism}

The Stokes vector $I=(I, Q, U, V)$ is defined by the components of the electromagnetic field (e.g. Chandrasekhar, 1960; Liou, 2002). It can be expressed with the real amplitudes, the phase difference and the angles $\beta$ and $\chi$ (Figure 3):

$$
\begin{aligned}
I & =a_{l}^{2}+a_{r}^{2}=I_{l}+I_{r} \\
Q & =a_{l}^{2}-a_{r}^{2}=I_{l}-I_{r}=I \cos 2 \beta \cos 2 \chi \\
U & =2 a_{l} a_{r} \cos \rho=I \cos 2 \beta \sin 2 \chi \\
V & =2 a_{l} a_{r} \sin \rho=I \sin 2 \beta
\end{aligned}
$$

The first Stokes parameter gives the total intensity, $Q$ indicates the degree of linear polarization, $U$ describes the plane of polarization and the ellipticity is given by $V$. For a

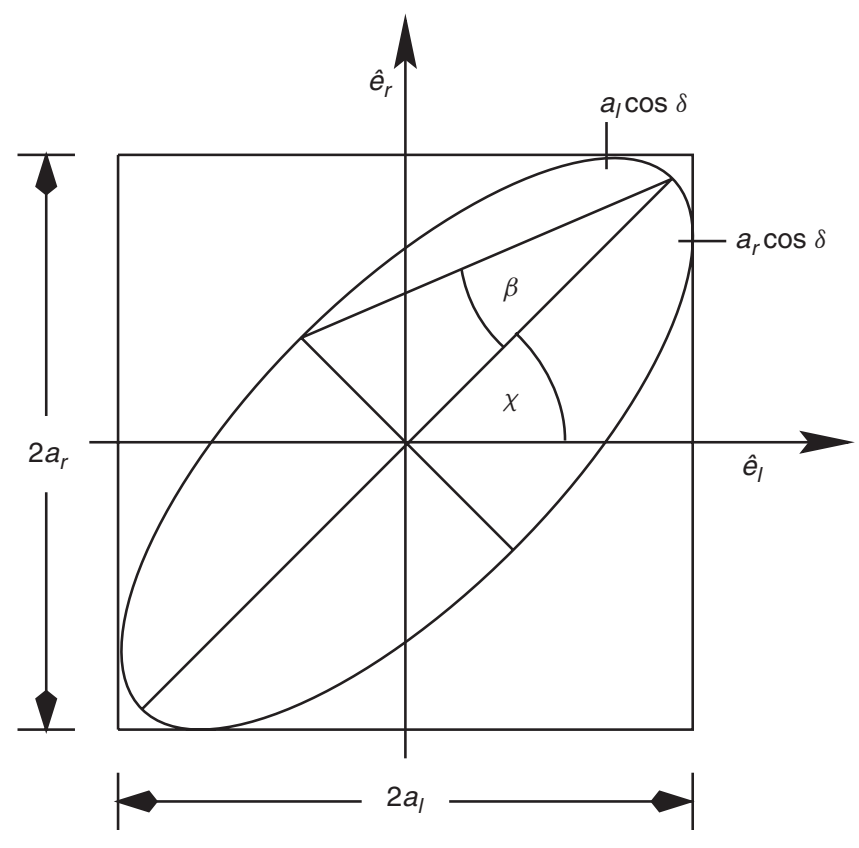

Figure 3 Illustration of an elliptically polarized electromagnetic wave 
single wave and fully polarized light $I^{2}=Q^{2}+U^{2}+V^{2}$ is valid. Natural radiation consists of the superposition of many different waves and contains a mixture of polarized and unpolarized light. In this case, the relation

$$
I^{2} \geq Q^{2}+U^{2}+V^{2}
$$

is valid. The degree of polarization is then given through:

$$
P=\frac{\sqrt{Q^{2}+U^{2}+V^{2}}}{I}
$$

\section{Transformation of Stokes Vectors and Global Coordinates}

Equations (18) describe the Stokes vector in local coordinates, which are used to describe single scattering processes. The radiative transfer equation refers to a Cartesian grid for the position and spherical coordinates for directions. Scattering processes change the direction of propagation and therefore a transformation between the local system, in which the scattering process is described, and the global coordinate system for the radiative transfer calculations is required. The geometry is illustrated in Figure 4. Assuming that the incident beam $P_{1}$ is scattered at the origin $O$ to $P_{2}$, the local coordinate system is defined in the plane $P_{1} O P_{2}$. Let $\boldsymbol{R}(\Theta)$ be the phase function and $\Theta$ the scattering angle in the local system. In order to apply the phase function the Stokes vector of the incident light has to

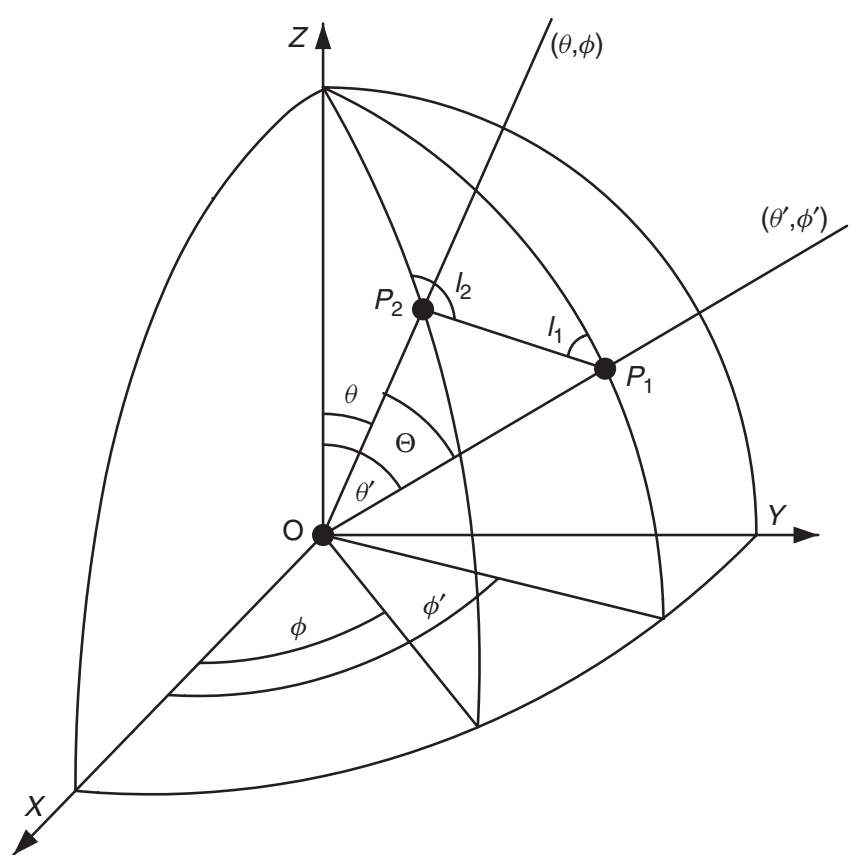

Figure 4 Directions of incoming and outgoing radiation with notation of angles be transformed from the directions to which the polarization refers in global coordinates (plane $P_{1} O Z$ ) to the local coordinates $\left(P_{1} O P_{2}\right)$. After performing the scattering process, a transformation from $P_{1} O P_{2}$ to $P_{2} O Z$ is necessary. The clockwise rotation angles for both rotations are $\left(-\iota_{1}\right)$ and $\left(\pi-\iota_{2}\right)$, respectively. The rotation transformation matrix for the Stokes vector reads:

$$
\boldsymbol{L}(\phi)=\left(\begin{array}{cccc}
1 & 0 & 0 & 0 \\
0 & \cos ^{2} \phi & \sin ^{2} \phi & 0 \\
0 & \sin ^{2} \phi & \cos ^{2} \phi & 0 \\
0 & 0 & 0 & 1
\end{array}\right)
$$

The scattered beam is computed from:

$$
\boldsymbol{I}(\theta, \phi)=\boldsymbol{L}\left(\pi-\iota_{2}\right) \boldsymbol{R}(\cos \Theta) \boldsymbol{L}\left(-\iota_{1}\right) \boldsymbol{I}\left(\theta^{\prime}, \phi^{\prime}\right)
$$

The phase function $\boldsymbol{P}\left(\theta, \phi ; \theta^{\prime}, \phi^{\prime}\right)$ is transformed from the local system to the global system by:

$$
\boldsymbol{P}\left(\theta, \phi ; \theta^{\prime}, \phi^{\prime}\right)=\boldsymbol{L}\left(\pi-\iota_{2}\right) \boldsymbol{R}(\cos \Theta) \boldsymbol{L}\left(-\iota_{1}\right)
$$

For a set of discrete angles $\left(\theta, \phi ; \theta^{\prime}, \phi^{\prime}\right)$, the angles $\iota_{1}$ and $\iota_{2}$ can be calculated with $\mu=\cos \theta$ and $\mu^{\prime}=\cos \theta^{\prime}$ :

$$
\cos \iota_{1}=\frac{-\mu+\mu^{\prime} \cos \Theta}{ \pm\left(1-\cos ^{2} \Theta\right)^{1 / 2}\left(1-\mu^{\prime 2}\right)^{1 / 2}}
$$

and

$$
\cos \iota_{2}=\frac{-\mu^{\prime}+\mu \cos \Theta}{ \pm\left(1-\cos ^{2} \Theta\right)^{1 / 2}\left(1-\mu^{2}\right)^{1 / 2}}
$$

The plus and minus signs in equations (24) and (25) are used for $\pi<\phi-\phi^{\prime}<2 \pi$ and $0<\phi-\phi^{\prime}<\pi$, respectively. The scattering angle $\Theta$, which is given in local coordinates, transforms to the global system through:

$$
\begin{aligned}
\cos \Theta & =\mu \mu^{\prime}+\left(1-\mu^{2}\right)^{1 / 2}\left(1-\mu^{\prime 2}\right)^{1 / 2} \cos \left(\phi-\phi^{\prime}\right) \\
& =\cos \theta \cos \theta^{\prime}+\sin \theta \sin \theta^{\prime} \cos \left(\phi-\phi^{\prime}\right)
\end{aligned}
$$

\section{Vector Radiative Transfer Equation}

Replacing the intensities in equation (14) by Stokes vectors leads to the Vector Radiative Transfer Equation (VRTE). As a consequence, scattering and extinction coefficients and the phase function become matrices. Following equation (14) the VRTE can be written in the general form:

$$
\begin{aligned}
\frac{\mathrm{d}^{3} \boldsymbol{I}(x, y, z, \theta, \phi)}{\frac{1}{\eta} \mathrm{d} x \frac{1}{\delta} \mathrm{d} y \frac{1}{\mu} \mathrm{d} z} & =-\boldsymbol{\sigma}_{\boldsymbol{e}}(x, y, z, \theta, \phi) \boldsymbol{I}(x, y, z, \theta, \phi) \\
& +\boldsymbol{\sigma}_{\boldsymbol{a}}(x, y, z, \theta, \phi) \boldsymbol{B}(T(x, y, z)) \\
+ & \int_{0}^{2 \pi} \int_{0}^{\pi} \boldsymbol{P}\left(x, y, z, \theta, \phi, \theta^{\prime}, \phi^{\prime}\right) \\
& \boldsymbol{I}\left(x, y, z, \theta^{\prime}, \phi^{\prime}\right) \sin \theta^{\prime} \mathrm{d} \theta^{\prime} \mathrm{d} \phi^{\prime}
\end{aligned}
$$


In general, the phase matrix $\boldsymbol{P}$ and the extinction matrix $\sigma_{e}$ are nondiagonal and couple the four components of the Stokes vector. Therefore, the differential equations do not decouple and have to be solved simultaneously. In the scattering phase matrix $\boldsymbol{P}$, three dimensions for position and space and four for the propagation directions of the incident and scattered radiation have to be considered. Therefore, $\boldsymbol{P}$ cannot be normalized and a volume scattering coefficient cannot be defined. As a consequence, the single scattering albedo $\omega_{0}$ as defined by equation (15) does not exist.

Another interesting fact to note is that there is no well-defined optical thickness for the four component Stokes vector. The exponential attenuation law (Bouguer-Lambert-Beer's law) for the transmission is not applicable and the transmission along path length $\mathrm{d} s$ cannot be calculated using:

$$
I(s+\mathrm{d} s)=I(s) e^{-\sigma_{e} \mathrm{~d} s}
$$

The differences in the Stokes vector components have to be computed at a series of small enough intervals $\mathrm{d} s$ to ensure that the change in intensity is linear in the path length coordinate:

$$
\mathrm{d} \boldsymbol{I}=\sigma_{e} \boldsymbol{I} \mathrm{d} s
$$

\section{SCATTERING AND PHASE FUNCTIONS}

It is one objective of scattering theory to quantify the scattering phase matrix, single scattering albedo, and the scattering cross sections for the individual scatterers, which can comprise molecules, aerosols, ice crystals, leafs, stems, and so on. The size parameter $\alpha_{\text {size }}=2 \pi r / \lambda$, which defines the relation between particle radius $r$ and wavelength $\lambda$, the complex refraction index and the particle shape are often used to determine the appropriate solution method.

In case of isotropic scattering, the phase function is given by $p(\cos \Theta)=1$. For many atmospheric applications, Rayleigh scattering is of particular interest. Lord Rayleigh first characterized it in 1871 in the context of the explanation for the blue sky. Although its original application focused on sunlight and atmospheric molecules, it was found that Rayleigh scattering theory could be applied to processes where the particle size is much smaller than the wavelength. As a rule of thumb, $\alpha_{\text {size }}$ should be smaller than 0.1. The phase function describing Rayleigh scattering can be formulated as:

$$
p(\cos \Theta)=\frac{3}{4}\left(1+\cos ^{2} \Theta\right)
$$

with the corresponding phase matrix:

$$
\boldsymbol{R}=\frac{3}{2}\left(\begin{array}{cccc}
\cos ^{2} \Theta & 0 & 0 & 0 \\
0 & 1 & 0 & 0 \\
0 & 0 & \cos \Theta & 0 \\
0 & 0 & 0 & \cos \Theta
\end{array}\right)
$$

Following Chandrasekhar (1960), the scattered intensity $I^{\text {scat }}$ can be written as:

$$
I^{\text {scat }}=\frac{128 \pi^{5}}{3 \lambda^{4}} \alpha_{p}^{2} I \mathrm{~d} \omega \frac{3}{4}\left(1+\cos ^{2} \Theta\right) \frac{\mathrm{d} \omega^{\prime}}{4 \pi}
$$

with $\alpha_{p}$ being the polarizability. The scattering coefficient per particle can be introduced as:

$$
\sigma_{s, p}=\frac{128 \pi^{5}}{3 \lambda^{4}} \alpha_{p}^{2}
$$

Figure 5 shows a Rayleigh scattering diagram in polar coordinates and a scattering angle versus scattering phase function representation. The three curves describe $I_{l}, I_{r}$, and $I_{l}+I_{r}$ (the scale is not identical in each case). Rayleigh scattering theory cannot be applied if the particle size is not small compared to the wavelength, for example, for interactions of natural light with cloud and rain droplets, aerosols, ice crystals, or vegetation canopies. For particles that are large compared to the wavelength, the geometric optics approach (or ray tracing technique) can be applied (e.g. Macke et al., 1996). For cases with particles sizes, which are comparable in size with the wavelength, exact solutions for single scattering problems exist only for highly symmetrical particle shapes if the surface of a particle is an iso-plane of a coordinate system, in which the vector wave equations can be derived. Under these conditions, the wave equation can be separated into a set of differential equations, which can be solved. Mie (1908) analyzed the problem for spheres in a spherical coordinate system using spherical harmonics. The solution for the scattering matrix and the scattering cross sections are obtained from an infinite series of Mie-coefficients, which are determined from the corresponding Legendre polynomials and spherical Bessel functions (Liou, 1992). A selection of Mie type phase functions is presented in Figure 6. The calculations are based on standard gamma drop size distributions (Hansen and Travis, 1974) characterized by effective radii of $10 \mu \mathrm{m}$ and $100 \mu \mathrm{m}$ for cloud water and rain, respectively. The wavelengths referred to in Figure 6 are $0.55 \mu \mathrm{m}$, $10 \mu \mathrm{m}$, and $1 \mathrm{~mm}$ for the visible, infrared, and microwave, respectively. For the visible and infrared wavelengths, the rain and water drops are comparably large and a strong forward peak characterizes the Mie scattering phase functions. In the infrared spectral region, the scattering phase function decreases monotonously with scattering angle. For the visible, a minimum around $105^{\circ}$ is obtained. Since the microwave wavelength is much larger than the cloud water droplet, Rayleigh scattering occurs. For nonspherical particles, which are rotationally symmetrical, numerical methods have been developed to extend the applicability of Mie theory (e.g. Mishchenko et al., 1996; Rother and Schmidt, 1996; Mishchenko et al., 2000). 

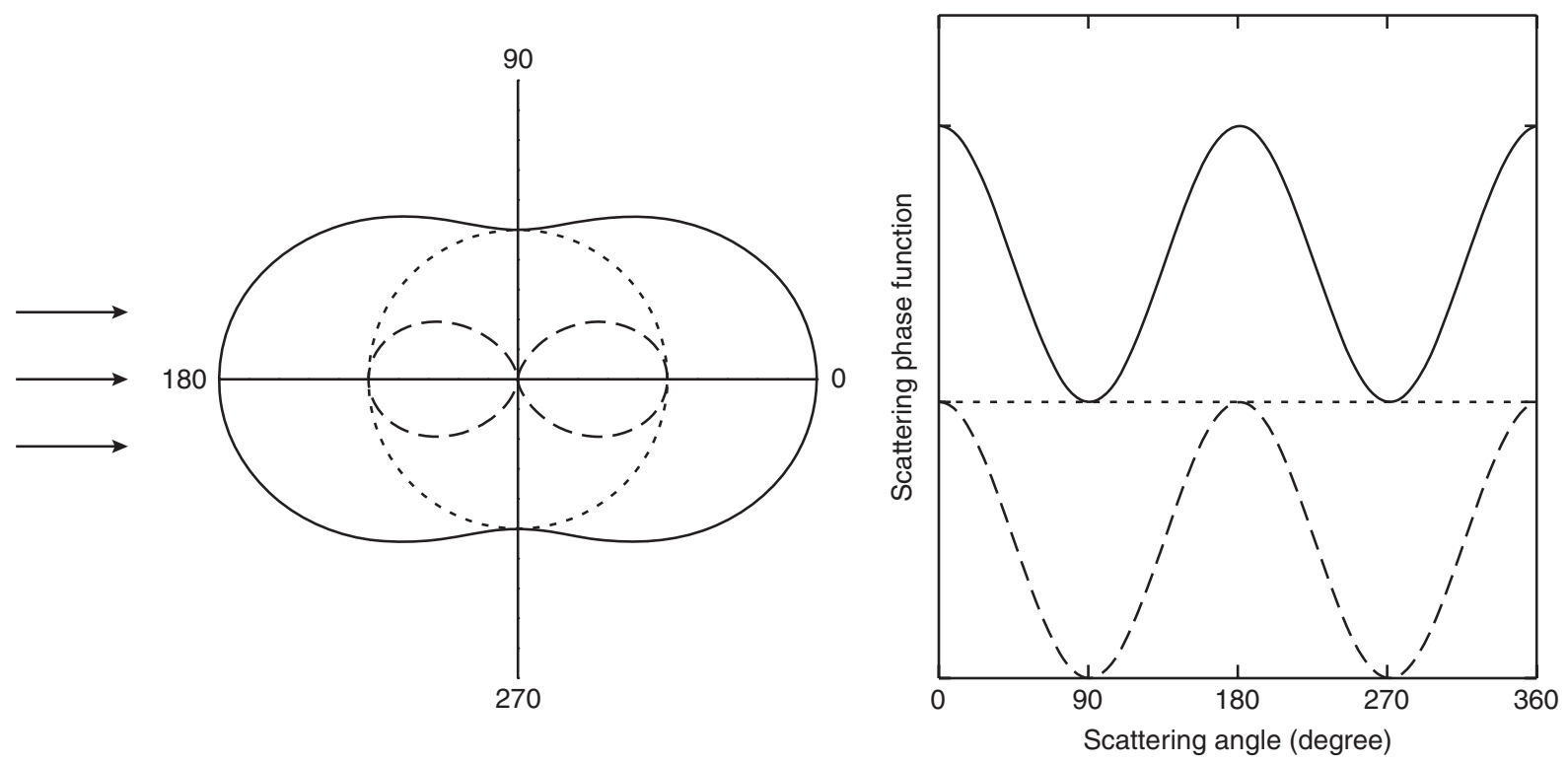

Figure 5 Schematic view of the Rayleigh phase function (after Goody and Yung, 1961)

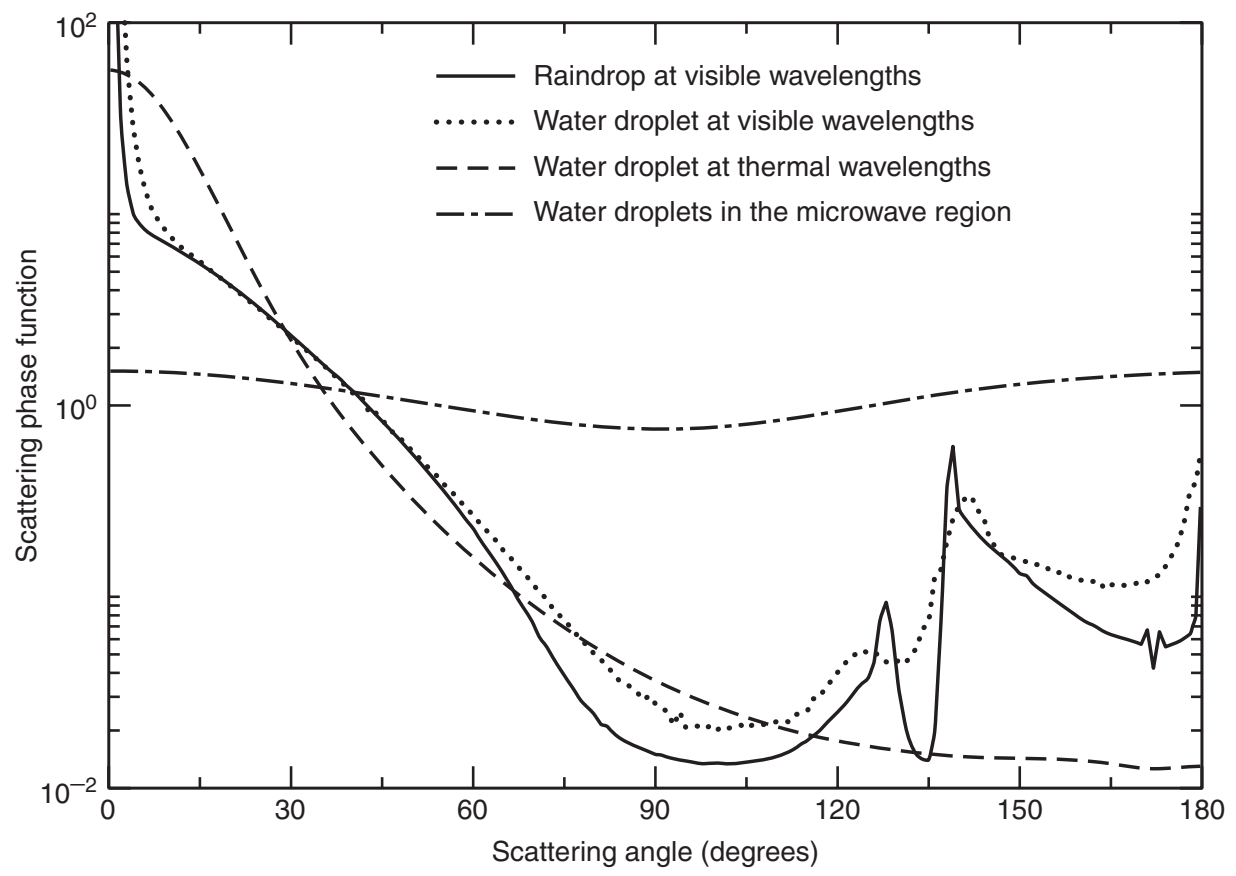

Figure 6 Scattering phase functions for visible $(0.55 \mu \mathrm{m})$, infrared $(10 \mu \mathrm{m})$, and microwave $(1 \mathrm{~mm})$ wavelengths. Effective radii of $10 \mu \mathrm{m}$ and $100 \mu \mathrm{m}$ for cloud water and rain characterize the drop size distributions

In order to model scattering of microwave radiation from vegetation canopies, randomly oriented nonspherical scatterers of finite size can be used. Circular discs are used to model deciduous vegetation (e.g. Tsang et al., 1981). Coniferous vegetation is described by needles (e.g. Eom and Fung, 1986) and stems and branches, which are represented as circular cylinders of finite size (e.g. Cohen et al., 1983; Karam and Fung, 1988). However, it has to be noted that in classical vector radiative transfer theory it is assumed that discrete particles scatter independently. This assumption is valid if the particles are distributed randomly and if the randomness of relative positions is comparable to or larger than the wavelength (Tsang et al., 1985). In dense and/or discontinuous media, which are 
characterized by clusters of scattering particles, collective scattering effects have to be included. Numerous models for the different wavelengths exist in the reviewed literature. Tsang et al. (1995) provide a brief review on different approaches and an application to passive microwave remote sensing.

\section{BOUNDARY CONDITIONS - THE SURFACE}

In general, natural surfaces reflect radiation anisotropically. The reflection at the surface can be described by the bidirectional reflectance function $\gamma$. "Bidirectional" refers to the fact that the reflection function depends on the direction of the incident radiation and the direction of the observation or outgoing radiation. The formal representation for $\gamma$ is (e.g. Mishchenko et al., 1999; Tsang et al., 1985):

$$
I(\theta, \phi)=\mu^{\prime} \gamma\left(\theta, \phi ; \theta^{\prime}, \phi^{\prime}\right) F
$$

with $\pi F$ the incident flux per unit area perpendicular to the incident beam (indices for polarization and frequency dependency are omitted). $\mu^{\prime} \gamma_{r, t}\left(\theta, \phi ; \theta^{\prime}, \phi^{\prime}\right)$ defines the bistatic scattering coefficient $\sigma_{r, t}^{o}(r$ and $t$ indicate the polarization), which satisfies the principle of reciprocity:

$$
\sigma_{r, t}^{o}\left(\theta, \phi ; \theta^{\prime}, \phi^{\prime}\right)=\sigma_{t, r}^{o}\left(\theta^{\prime}, \phi^{\prime} ; \theta, \phi\right)
$$

The emissivity $\varepsilon_{r}$ and reflectivity $\Gamma_{r}$ are given by:

$$
\varepsilon_{r}\left(\theta^{\prime}, \phi^{\prime}\right)=1-\Gamma_{r}\left(\theta^{\prime}, \phi^{\prime}\right)
$$

and

$$
\begin{aligned}
\Gamma_{r}\left(\theta^{\prime}, \phi^{\prime}\right)= & \frac{1}{4 \pi \mu} \int_{0}^{2 \pi}\left(\sigma_{r r}^{o}\left(\theta, \phi, \theta^{\prime}, \phi^{\prime}\right)\right. \\
& \left.+\sigma_{t r}^{o}\left(\theta, \phi, \theta^{\prime}, \phi^{\prime}\right)\right) \sin \theta d \theta d \phi .
\end{aligned}
$$

The reflected intensity $I^{S}$ at polarization $r$ can be expressed as:

$$
\begin{aligned}
I_{r}^{s}\left(\theta^{\prime}, \phi^{\prime}\right)= & \frac{1}{4 \pi \mu} \int_{0}^{2 \pi}\left(\sigma_{r r}^{o}\left(\theta, \phi, \theta^{\prime}, \phi^{\prime}\right) I_{r}(\theta, \phi)\right. \\
& \left.+\sigma_{t r}^{o}\left(\theta, \phi, \theta^{\prime}, \phi^{\prime}\right) I_{t}(\theta, \phi)\right) \sin \theta \mathrm{d} \theta \mathrm{d} \phi
\end{aligned}
$$

Since many remote sensing, engineering, and biophysical applications rely on an accurate description of the bidirectional reflection function or the bistatic scattering coefficient the number of models, which relate geophysical surface parameters to both quantities, is large. The concepts and complexities vary depending on the application, for which they were designed. A number of review articles or overviews on the different models and frequency ranges exist, for example, Myneni et al. (1989), Goel (1988), Snyder and Wan (1998), Kerr and Wigneron (1995).

\section{APPLICATION TO PASSIVE MICROWAVE RADIOMETRY}

Equation (27) is the most general form for vector RTT. For many applications simplifications can be made to obtain analytical solutions. As an example, we will derive a solution for equation (27) for passive microwave applications. The number of geophysical parameters, which can be derived from passive microwave observations, is large and covers a wide range of applications (Ulaby et al., 1982): (1) oceans (surface wind speed, temperature, salinity, and oil spills; sea ice extent and age), (2) meteorology (temperature profile, water vapor profile, liquid water (clouds and rain), and integrated water vapor), and (3) agriculture and hydrology (soil moisture distribution, flood mapping, delineation of freeze-thaw boundaries, snow cover extent, snow water equivalent, snow wetness, and continental ice sheets). The derivation outlined in the following paragraphs has been used as the basis for a number of applications, especially for the land surface (e.g. Drusch et al., 2001; Kerr and Njoku, 1990; Mo et al., 1982).

For plane parallel azimuthally isotropic and horizontally homogeneous atmospheres, the radiances become a function of $z$ and $\theta$. Owing to the azimuthally symmetry, there is no interaction between the first two components and the last two components of the Stokes vector and total intensity and linear polarization can be derived without considering the $U$ and $V$ components of the Stokes vector. From equation 27, one obtains (Tsang et al., 1985):

$$
\begin{aligned}
\mu \frac{\mathrm{d} \boldsymbol{I}(z, \theta)}{\mathrm{d} z}=- & \boldsymbol{\sigma}_{\mathbf{e}}(z, \theta) \boldsymbol{I}(z, \theta)+\boldsymbol{\sigma}_{\mathbf{a}}(z, \theta) \boldsymbol{B}(T(z)) \\
& +\int_{0}^{\pi} \boldsymbol{P}\left(z, \theta, \theta^{\prime}\right) \boldsymbol{I}\left(z, \theta^{\prime}\right) \sin \theta^{\prime} \mathrm{d} \theta^{\prime}
\end{aligned}
$$

which is equivalent to:

$$
\begin{aligned}
& \cos \theta \frac{d}{\mathrm{~d} z}\left[\begin{array}{l}
I_{v}(z, \theta) \\
I_{h}(z, \theta)
\end{array}\right] \\
& =-\sigma_{e}(z)\left[\begin{array}{l}
I_{v}(z, \theta) \\
I_{h}(z, \theta)
\end{array}\right]+\sigma_{a}(z)\left[\begin{array}{c}
\frac{B(T)}{2} \\
\frac{B(T)}{2}
\end{array}\right] \\
& +\int_{0}^{\pi}\left[\begin{array}{ll}
P_{v v}\left(z, \theta, \theta^{\prime}\right) & P_{v h}\left(z, \theta, \theta^{\prime}\right) \\
P_{h v}\left(z, \theta, \theta^{\prime}\right) & P_{h h}\left(z, \theta, \theta^{\prime}\right)
\end{array}\right] \\
& {\left[\begin{array}{l}
I_{v}(z, \theta) \\
I_{h}(z, \theta)
\end{array}\right] \sin \theta^{\prime} \mathrm{d} \theta^{\prime}}
\end{aligned}
$$


The sources of radiation can be summarized as:

$$
\begin{aligned}
J(z, \mu)= & \frac{\sigma_{a}}{\sigma_{e}} \frac{B(T(z))}{2}+\omega_{0}(z) \int_{-1}^{1}\left(p_{r v}\left(z, \mu, \mu^{\prime}\right) I_{v}\left(z, \mu^{\prime}\right)\right. \\
& \left.+p_{r h}\left(z, \mu, \mu^{\prime}\right) I_{h}\left(z, \mu^{\prime}\right)\right) d \mu^{\prime}
\end{aligned}
$$

with the normalized phase functions $p$, which are obtained through:

$$
p_{r, t}=\frac{1}{\sigma_{s}} P_{r, t}
$$

Introducing the height of the atmosphere $z_{A}$ and the atmospheric optical depth $\delta$ :

$$
\begin{aligned}
\delta(z) & =\int_{z}^{z_{A}} \sigma_{e}\left(z^{\prime}\right) \mathrm{d} z^{\prime} \\
\mathrm{d} \delta & =-\sigma_{e} \mathrm{~d} z
\end{aligned}
$$

the radiative transfer equation for polarized radiation can be written as:

$$
\mu \frac{d}{\mathrm{~d} \delta} I_{r}(\delta, \mu)=I_{r}(\delta, \mu)-J_{r}(\delta, \mu)
$$

which is equivalent to equation (14). A formal solution for this expression can be obtained by separating the upward and downward intensities $I^{+}(\mu>0)$ and $I^{-}(\mu<0)$ :

$$
\begin{aligned}
I_{r}^{+}(\delta, \mu)= & I_{r}^{+}\left(\delta_{A}, \mu\right) \exp \left[-\frac{\delta_{A}-\delta}{\mu}\right] \\
& +\int_{\delta}^{\delta_{A}} J_{r}\left(\delta^{\prime}, \mu\right) \exp \left[-\frac{\delta^{\prime}-\delta}{\mu}\right] \frac{\mathrm{d} \delta^{\prime}}{\mu}
\end{aligned}
$$

and

$$
\begin{aligned}
I_{r}^{-}(\delta, \mu)= & I_{r}^{-}(0, \mu) \exp \left[-\frac{\delta}{|\mu|}\right] \\
& +\int_{0}^{\delta} J_{r}\left(\delta^{\prime}, \mu\right) \exp \left[-\frac{\delta-\delta^{\prime}}{|\mu|}\right] \frac{\mathrm{d} \delta^{\prime}}{|\mu|}
\end{aligned}
$$

If we assume a nonscattering atmosphere $\left(\omega_{0}=0\right.$ and $\left.\sigma_{a} / \sigma_{e}=1\right)$ and apply the Rayleigh-Jeans-Approximation (long wavelength limit, the emission by the earth's atmosphere is directly proportional to the temperature) equations (45) and (46) can be written as:

$$
\begin{aligned}
T_{\mathrm{Br}}^{+}(\delta, \mu)= & T_{\mathrm{Br}}^{+}\left(\delta_{A}, \mu\right) \exp \left[-\frac{\delta_{A}-\delta}{\mu}\right] \\
& +\int_{\delta}^{\delta_{A}} T\left(\delta^{\prime}\right) \exp \left[-\frac{\delta^{\prime}-\delta}{\mu}\right] \frac{\mathrm{d} \delta^{\prime}}{\mu}
\end{aligned}
$$

and

$$
\begin{aligned}
T_{\mathrm{Br}}^{-}(\delta, \mu)= & T_{\mathrm{Br}}^{-}(0, \mu) \exp \left[-\frac{\delta}{|\mu|}\right] \\
& +\int_{0}^{\delta} T\left(\delta^{\prime}\right) \exp \left[-\frac{\delta-\delta^{\prime}}{|\mu|}\right] \frac{\mathrm{d} \delta^{\prime}}{|\mu|}
\end{aligned}
$$

with $T_{\mathrm{Br}}$ being the equivalent Rayleigh-Jeans brightness temperature defined as:

$$
I=I_{v}+I_{h}=k_{B} v^{2} c^{2}\left(T_{B v}+T_{B h}\right)
$$

It should be noted that for several applications it is necessary to calculate the brightness temperatures by inversion of the Planck function to avoid systematic errors. For the sake of simplicity, we focus on the downward radiation at the bottom of the atmosphere $\left(\delta=\delta_{A}\right.$, e.g. the total optical depth of the atmosphere), and on the upward radiation at the top of the atmosphere $(\delta=0)$ as observed from a satellite. From equation (47) and (48), we obtain:

$$
\begin{aligned}
T_{\mathrm{Br}}^{+}(0, \mu)= & T_{\mathrm{Br}}^{+}\left(\delta_{A}, \mu\right) \exp \left[-\frac{\delta_{A}}{\mu}\right] \\
& +\int_{0}^{\delta_{A}} T(\delta) \exp \left[-\frac{\delta}{\mu}\right] \frac{\mathrm{d} \delta}{\mu} \\
= & T_{\mathrm{Br}}^{+}\left(\delta_{A}, \mu\right) \exp \left[-\frac{\delta_{A}}{\mu}\right]+\mathrm{T}_{\mathrm{BAup}}
\end{aligned}
$$

and

$$
\begin{aligned}
T_{\mathrm{Br}}^{-}\left(\delta_{A}, \mu\right)= & T_{\mathrm{Br}}^{-}(0, \mu) \exp \left[-\frac{\delta_{A}}{|\mu|}\right] \\
& +\int_{0}^{\delta_{A}} T(\delta) \exp \left[-\frac{\delta_{A}-\delta}{|\mu|}\right] \frac{\mathrm{d} \delta}{|\mu|} \\
= & T_{\mathrm{BSp}} \exp \left[-\frac{\delta_{A}}{|\mu|}\right]+T_{\mathrm{Badw}}
\end{aligned}
$$

with $T_{\mathrm{BAup}}, T_{\mathrm{Badw}}$, and $T_{\mathrm{BSp}}$ being the atmospheric upward, atmospheric downward, and space contribution, respectively. Since scattering in the atmosphere is neglected these contributions are not polarized. The upward radiation at the bottom of the atmosphere/top of the vegetation, $T_{\mathrm{Br}}{ }^{+}\left(\delta_{A}, \mu\right)$, needs further consideration, since this part contains the contribution from the surface. In order to determine $T_{\mathrm{Br}}{ }^{+}\left(\delta_{A}, \mu\right)$ the radiative transfer equation has to be solved for the canopy layer. One way to obtain $T_{\mathrm{Br}}{ }^{+}\left(\delta_{A}, \mu\right)$ is to replace the corresponding atmospheric parameters by the canopy optical depth $\delta_{c}$ and single scattering albedo $\omega_{c}$ in equation (44). Let the phase function describing scattering in the canopy layer being normalized as:

$$
\frac{1}{2} \int_{-1}^{1} P\left(\delta_{c}, \mu, \mu^{\prime}\right) \mathrm{d} \mu^{\prime}=1
$$


For vegetation fields with long cylindrical structures the scattering in the forward direction is the dominant part (Chuang et al., 1980). Consequently, the forward scattering can be expressed as the sum of a Dirac- $\delta$-function and a phase function $P^{*}\left(\mu, \mu^{\prime}\right)$, which is not peaked in the forward direction (Joseph et al., 1976):

$$
P\left(\delta_{c}, \mu, \mu^{\prime}\right)=2 \alpha \delta\left(\mu-\mu^{\prime}\right)+(1-\alpha) P^{*}\left(\mu, \mu^{\prime}\right)
$$

It should be noted that it is assumed that the phase function and the single scattering albedo are independent of $\delta_{c}$ in this approach. Different radiative transfer models for vegetation layers are available. An overview can be found in Kerr and Wigneron (1995). However, Joseph et al. (1976) showed that equation (44) is invariant in form when the phase function as defined in equation (53) is used. $\delta_{c}$, $\omega_{c}$ and $P\left(\delta_{c}, \mu, \mu^{\prime}\right)$ have to be replaced by $\delta_{c}{ }^{*}, \omega_{c}{ }^{*}$ and $P^{*}\left(\mu, \mu^{\prime}\right)$ with:

$$
\begin{aligned}
\delta_{c}^{*} & =\left(1-\alpha \omega_{c}\right) \delta \\
\omega_{c}^{*} & =\frac{(1-\alpha) \omega_{c}}{1-\alpha \omega}
\end{aligned}
$$

Under the assumption that forward scattering is the dominant process, and since $P^{*}\left(\mu, \mu^{\prime}\right)$ is not well known, this quantity is set to 0 . Little calculus yields the upward and downward contributions for the radiation at the top and the bottom of the canopy layer, respectively (Mo et al., 1982):

$$
\begin{aligned}
T_{\mathrm{CBr}}^{+}(0, \mu)= & T_{\mathrm{CBr}}^{+}\left(\delta_{c}^{*}, \mu\right) \exp \left[-\frac{\delta_{c}^{*}}{\mu}\right] \\
& +\left(1-\omega_{c}^{*}\right) T_{c}\left(1-\exp \left[-\frac{\delta_{c}^{*}}{\mu}\right]\right)
\end{aligned}
$$

and

$$
\begin{aligned}
T_{\mathrm{CBr}}^{-}\left(\delta_{c}^{*}, \mu\right)= & T_{\mathrm{CBr}}^{-}(0, \mu) \exp \left[-\frac{\delta_{c}^{*}}{\mu}\right] \\
& +\left(1-\omega_{c}^{*}\right) T_{c}\left(1-\exp \left[-\frac{\delta_{c}^{*}}{\mu}\right]\right)
\end{aligned}
$$

with the canopy temperature $T_{c}$. The downward radiation at the top of the canopy $T_{\mathrm{CBr}}{ }^{-}(0, \mu)$ is identical to the downward radiation at the bottom of the atmosphere $T_{\mathrm{Br}}{ }^{-}\left(\delta_{A}, \mu\right)$ as given in equation (51). The upward radiation at the bottom of the atmosphere $T_{\mathrm{Br}}{ }^{+}\left(\delta_{A}, \mu\right)$ is given by equation (55). Combining (56) with (51) and (55) with (50) gives the upward radiation at the top of the atmosphere $T_{\mathrm{Br}}{ }^{+}(0, \mu)$ and the downward radiation at the bottom of the vegetation layer $T_{\mathrm{CBr}}{ }^{-}\left(\delta_{c}{ }^{*}, \mu\right)$ :

$$
\begin{gathered}
T_{\mathrm{CBr}}^{-}\left(\delta_{c}^{*}, \mu\right)=\left(T_{\mathrm{BSp}} \exp \left[-\frac{\delta_{A}}{|\mu|}\right]+T_{\mathrm{Badw}}\right) \\
\exp \left[-\frac{\delta_{c}^{*}}{|\mu|}\right]+\left(1-\omega_{c}^{*}\right) T_{c}\left(1-\exp \left[-\frac{\delta_{c}^{*}}{|\mu|}\right]\right) \\
T_{\mathrm{Br}}^{+}(0, \mu)=\left(T_{\mathrm{CBr}}^{+}\left(\delta_{c}^{*}, \mu\right) \exp \left[-\frac{\delta_{c}^{*}}{\mu}\right]+\left(1-\omega_{c}^{*}\right)\right. \\
\left.T_{c}\left(1-\exp \left[-\frac{\delta_{c}^{*}}{\mu}\right]\right)\right) \exp \left[-\frac{\delta_{A}}{\mu}\right]+T_{\mathrm{BAup}}
\end{gathered}
$$

Equations (57) and (58) are coupled through the surface interaction where a portion of the downward radiation at the bottom of the canopy layer is reflected. $T_{\mathrm{CBr}}{ }^{+}\left(\delta_{c}{ }^{*}, \mu\right)$ can be written as:

$$
\begin{aligned}
T_{\mathrm{CBr}}^{+}\left(\delta_{c}^{*}, \mu\right)= & (1-\Gamma(\mu)) T_{S} \\
& +\frac{1}{2 \mu} \int_{-1}^{0}\left[\sigma_{r r}^{*}\left(\mu, \mu^{\prime}\right) T_{\mathrm{CBr}}^{-}\left(\delta_{c}^{*}, \mu^{\prime}\right)\right. \\
& \left.+\sigma_{r t}^{*}\left(\mu, \mu^{\prime}\right) T_{\mathrm{CBt}}^{-}\left(\delta_{c}^{*}, \mu^{\prime}\right)\right] \mathrm{d} \mu^{\prime}
\end{aligned}
$$

with $T_{S}$ the surface temperature and

$$
\begin{aligned}
\Gamma_{r}(\mu) & =\frac{1}{2 \mu} \int_{0}^{1}\left[\sigma_{r r}^{*}\left(\mu^{\prime}, \mu\right)+\sigma_{t r}^{*}\left(\mu^{\prime}, \mu\right)\right] \mathrm{d} \mu^{\prime} \\
\sigma_{t r}^{*}\left(\mu^{\prime}, \mu\right) & =\frac{1}{2 \pi} \int_{0}^{2 \pi} \sigma_{t r}^{o}\left(\mu^{\prime}, \mu, \phi-\phi^{\prime}\right) \mathrm{d} \phi
\end{aligned}
$$

In the case of specular reflection, the bistatic scattering coefficients for cross polarization (subscripts $r t$ or $t r$ ) are 0 . For $r=t, \sigma *$ is given as:

$$
\sigma_{r t}^{*}\left(\mu^{\prime}, \mu\right)=2 \mu \sigma_{r}^{\text {Spec }} \boldsymbol{\delta}\left(\mu_{s}-\mu\right)
$$

The reflected radiation can then be written as:

$$
T_{\mathrm{CBr}}^{+}\left(\delta_{c}^{*}, \mu\right)=(1-\Gamma(\mu)) T_{S}+\Gamma(\mu) T_{\mathrm{CBr}}^{-}\left(\delta_{c}^{*}, \mu\right)
$$

For the computation of $\Gamma(\mu)$, which is based on the dielectric properties of the soil, various methods do exist (e.g. Kerr and Njoku, 1990; Wilheit, 1978). Generally, the reflection of the land surface is not specular. To take into account roughness effects, the specular reflectivities can be modified, for example, according to Choudhury et al. (1979) or Wegmüller and Mätzler (1999). A comprehensive and detailed discussion on passive microwave remote sensing of land surfaces can be found in Choudhury et al. (1995). 


\section{SOLUTIONS}

The radiative transfer problem as outlined above is rather complex, and general analytic solutions of the vector radiative transfer equation, which provide the spatial distribution of intensities, do not exist. Depending on the application several approximations may apply and a number of methods to numerically solve the RTT have been developed. Often the approximations cannot resolve the full angular dependence and/or the full polarization information. Furthermore, most solutions are achieved for the one-dimensional problem where only vertical variations are considered and the atmosphere/surface is assumed to be horizontally homogeneous. Additional geometrical complications arise from the sphericity of the Earth's atmosphere. In the case of a nonscattering and horizontally homogeneous atmosphere, the RTT reduces to a relatively simple form (47) and (48), which can easily be solved by numerical integration when the vertical distribution of the absorption coefficient and temperature is known. Because the ratio between the size of the scattering particle and the wavelength determines the scattering efficiency, the neglect of scattering is well justified at microwave wavelengths, except when (large) precipitation drops are present. At short wavelengths, for example, solar radiation, scattering by atmospheric gases needs to be considered. Detailed descriptions of methods to solve the RTT can be found in many text books and publications. Following Lenoble (1985), the different methods have been separated in three groups: (1) exact analytical methods, (2) computational methods, and (3) approximate methods. This section briefly introduces and discusses some of the different concepts. According to Lenoble (1985), the primary usefulness of exact analytical methods is the understanding of the mathematical structure and of the general behavior of solutions of the radiative transfer equation. We focus on computational and approximate methods, which are most common in hydrological and meteorological applications.

Computational methods can take into account the real characteristics of the atmosphere. The accuracy of the results depends on the computer and the computation time. Monte Carlo methods simulate the path of individual photons, which experience absorption and scattering according to any given phase matrix within the medium of interest. All radiation variables including polarization can be derived at any point by averaging many photons propagating statistically within the considered domain. Although the computational cost is relatively high, one of the benefits of the Monte Carlo method is the ability to consider complex 3D geometries including broken clouds. The Monte Carlo technique offers a straightforward interpretation of the radiation field, which allows controlling the accuracy by the number of photons considered. Therefore, the Monte Carlo method is often used as a reference to evaluate other models. The Monte Carlo method can be formulated either as a forward or backward problem depending on the application. For example, if remote sensing applications are considered it is useful to consider the photons arriving at the detector and trace their origin backwards. The main disadvantages are (1) the statistical fluctuation in the results and (2) the limitation to optical depths below 100.

In the Discrete Ordinate Method (DOM, Chandrasekhar, 1960), the angular dependency of the radiative transfer equation is discretized and the solution consists of a set of first-order differential equations. DISORT (Stamnes et al., 1988) is a numerically implemented solution for vertically inhomogeneous, layered media. It solves the RTT for a scattering, absorbing, and emitting medium with an arbitrarily specified bidirectional reflectivity at the lower boundary. Primary advantages of the DOM are: (1) the solution of the radiative transfer equation can be derived explicitly and the intensity calculations do not depend on the total optical depth of cloud or aerosol layers. (2) Analytic two-stream and four-stream solutions can be derived in closed forms. (3) Computational times are relatively small compared to other techniques. Polarization effects are not included in the calculations.

The Spherical Harmonical Discrete Ordinate Method (SHDOM) calculates nonpolarized monochromatic or spectral band radiative transfer in a one, two, or threedimensional medium for either collimated solar and/or thermal emission sources of radiation (Evans, 1998). The program package is freely available from http://nit.colo rado.edu/ evans/shdom.html. In contrast to the standard DOM, the angular part of the source function is represented with a spherical harmonic expansion, which allows a more efficient calculation of the scattering integral.

Within the Successive Order of Scattering (SOS) method, multiple scattering is treated as a sequence of single scattering events. In order to fulfill this approximation, the medium has to be divided into optically thin layers, which is appropriate in most microwave applications. SOS gives a better understanding of the problem than many of the other methods, because the photon is followed at each scattering process. Again, polarization effects cannot be taken into account. Another disadvantage is the high computational cost for situations with very weak absorption and high optical depths.

The adding, doubling, and matrix operator methods (Plass, 1973) are closely related. The reflection and transmission functions of an initial, optically thin sub layer are obtained by single scattering calculations for all incident and emergent angles at once. This layer is successively doubled until the given optical thickness of the homogeneous layer is reached. Then the homogeneous layers are added to an inhomogenous atmosphere. Polarization can be included in this approach.

Approximate methods include either an approximation of the real atmosphere or of the radiative transfer problem. 
The applicability of approximate methods depends primarily on the required accuracy. The two-stream-approximation is commonly implemented in numerical weather prediction and climate models to calculate heating rates in the atmosphere and the Earth surface. For this purpose, it is sufficient to derive radiant fluxes rather than intensities, which reduces the computational effort. Since radiative fluxes are angular averaged properties, details of the angular intensity variation can be neglected. The strategy is to introduce an effective angular averaged intensity (stream) and determine an effective scattering angle. It is equivalent to the lowest order $(\mathrm{N}=1)$ of the Discrete Ordinate Method. Considering the upward and downward components (two streams) leads to a pair of coupled, first-order differential equations, the two-stream equations. The coupling between the differential equations depends on the way the intensity and phase function are approximated (e.g. Liou, 2002). Within a homogeneous medium, the two-stream equations can be solved analytically using standard methods; for anisotropic scattering, a number of methods have been proposed. The two-stream-approximation is most accurate under isotropic conditions when the mean inclination (average cosine) is the same in both directions but works reasonable at the boundaries, too. The Eddington-approximation originally developed for studying the radiative equilibrium in stellar atmospheres has a similar application range as the two-stream-approximation. Here the angular dependence is approximated by a polynom resulting in a similar set of equations as for the two-stream approximation. Both approximations can be improved by implementing the Dirac function for a better representation of the forward scattering peak in the phase function and are then referred to as $\delta$-twostream or $\delta$-Eddington. Differences between the Eddingtonand two-stream method can be only due to the choice of the mean inclination, the boundary conditions and the choice of the phase function (Thomas and Stamnes, 1999).

\section{Acknowledgments}

The authors would like to thank Dr. H. Czekala (Radiometer Physics $\mathrm{GmbH}$ ) for many helpful discussions and providing Figures 2, 3, and 4. Prof. A. Macke (Institute for Marine Sciences Kiel) provided Figure 6 and suggestions on the original manuscript. The paper benefited from many comments from Prof. Simmer and Dr. J. Schulz (both Meteorological Institute Bonn), Dr. P. Bauer and Dr. R. Engelen (both ECMWF), and an anonymous reviewer. We would like to thank R. Hine (ECMWF) for his help with the final preparation of the figures.

\section{REFERENCES}

Chandrasekhar S. (1960) Radiative Transfer, Dover Publications: New York, p. 303.
Choudhury B.J., Kerr Y.H., Njoku E.G. and Pampaloni P. (Eds.) (1995) Passive Microwave Remote Sensing of Land-Atmosphere Interactions, VSP Utrecht: The Netherlands, p. 685.

Choudhury B.J., Schmugge T.J., Chang A. and Newton R.W. (1979) Effect of surface roughness on the microwave emission from soils. Journal of Geophysical Research, 84, 5699-5706.

Chuang S.L., Kong J.A. and Tsang L. (1980) Radiative transfer theory for passive microwave remote sensing of a two-layer random medium with cylindrical structures. Journal of Applied Physics, 51, 5588-5593.

Cohen L.D., Haracz R.D., Cohen A. and Acquista C. (1983) Scattering of light from arbitrarily oriented finite cylinders. Applied Optics, 23, 436-441.

Drusch M., Wood E.F. and Jackson T.J. (2001) Vegetative and atmospheric corrections for the soil moisture retrieval from passive microwave remote sensing data: results from the Southern Great Plains hydrology experiment 1997. Journal of Hydrometeorology, 2, 181-192.

Eom H.J. and Fung A.K. (1986) Scattering from a random layer embedded with dielectric needles. Remote Sensing of Environment, 19, 139-149.

Evans K.F. (1998) The spherical harmonic discrete ordinate method for three-dimensional atmospheric radiative transfer. Journal of the Atmospheric Sciences, 55, 429-446.

Goel N.S. (1988) Models of vegetation canopy reflectance and their use in estimation of biophysical parameters from reflectance data. Remote Sensing of Environment, 4, 1-222.

Goody R.M. and Yung Y.L. (1961) Atmospheric Radiation, Oxford University Press: Oxford, p. 519.

Hansen J.E. and Travis L.D. (1974) Light scattering in planetary atmospheres. Space Science Reviews, 16, 527-610.

Joseph J.H., Wiscombe W.J. and Weinman J.A. (1976) The DeltaEddington approximation for radiative flux transfer. Journal of the Atmospheric Sciences, 33, 2452-2459.

Karam M.A. and Fung A.K. (1988) Electromagnetic scattering from a layer of finite length, randomly oriented, dielectric, circular cylinders over a rough interface with application to vegetation. International Journal of Remote Sensing, 9, 1109-1134.

Kerr Y.H. and Njoku E.G. (1990) A semi-empirical model for interpreting microwave emission from semiarid land surfaces as seen from space. IEEE Transactions on Geoscience and Remote Sensing, 28, 384-393.

Kerr Y.H. and Wigneron J.P. (1995) Vegetation models and observations: a review. Passive Microwave Remote Sensing of Land-Atmosphere Interactions, VSP: Utrecht, pp. 317-344.

Lean J. and Rind D. (1998) Climate forcing by changing solar radiation. Journal of Climate, 11, 3069-3094.

Lenoble J. (1985) Radiative Transfer in Scattering and Absorbing Atmospheres: Standard Computational Procedures, A. Deepak Publishing: Hampton, p. 300.

Liou K.N. (1992) Radiation and Cloud Processes in the Atmosphere, Oxford University Press: Oxford, p. 487.

Liou K.N. (2002) An Introduction to Atmospheric Radiation, International Geophysics Series, Second Edition, Vol. 84, Academic Press: p. 583.

Macke A., Mishchenko M.I. and Cairns B. (1996) The influence of inclusions on light scattering by large ice particles. Journal of Geophysical Research, 101, 23311-23316. 
Mie G. (1908) Beiträge zur optik trüber medien, speziell kolloidaler metallösungen. Annalen der Physik, 25, 377-445.

Mishchenko M.I., Dlugach J.M., Yanovitskij E.G. and Zakharova N.T. (1999) Bidirectional reflectance of flat, optically thick particulate layers: an efficient radiative transfer solution and applications to snow and soil surfaces. Journal of Quantitative Spectroscopy, 63, 409-432.

Mishchenko M.I., Hovenier J.W. and Travis L.D. (Eds.) (2000) Light Scattering by Nonspherical Particles. Theory, Measurements, and Applications, Academic Press: San Diego.

Mishchenko M.I., Travis L.D. and Mackowski D.W. (1996) T-matrix computations of light scattering by non-spherical particles: a review. Journal of Quantitative Spectroscopy \& Radiative Transfer, 55, 535-575.

Mo T., Choudhury B.J., Schmugge T.J., Wang J.R. and Jackson T.J. (1982) A model for microwave emission from vegetation - covered fields. Journal of Geophysical Research, 87, 11229-11237.

Myneni R.B., Ross J. and Asrar G. (1989) A review on the theory of photon transport in leaf canopies. Agricultural and Forest Meteorology, 45, 1-153.

Plass G.N. (1973) Matrix operator theory of radiative transfer: 1. Rayleigh scattering. Applied Optics, 12, 314-329.

Rother T. and Schmidt K. (1996) The discrete mie-formalism for plane-wave scattering on axisymmetric particles. Journal of Electromagnetic Waves and Applications, 10, 273-297.
Salby M.L. (1996) Fundamentals of Atmospheric Physics, Academic Press: London.

Snyder W.C. and Wan Z. (1998) BRDF models to predict spectral reflectance and emissivity in the thermal infrared. IEEE Transactions on Geoscience and Remote Sensing, 36, 214-225.

Stamnes K., Tsay S.C., Wiscombe W. and Jayaweera K. (1988) A numerically stable algorithm for discrete-ordinate-method radiative transfer in multiple scattering and emitting layered media. Applied Optics, 27, 2502-2509.

Thomas G.E. and Stamnes K. (1999) Radiative Transfer in the Atmosphere and Ocean, Dessler A.J., Hughton J.T. and Rycroft M.J. (Eds.), Cambridge University Press: p. 517.

Tsang L., Kong J.A. and Shin R.T. (1985) Theory of Microwave Remote Sensing, John Wiley \& Sons: New York, p. 613.

Tsang L., Kubacsi M.C. and Kong J.A. (1981) Radiative transfer theory for active remote sensing of a layer of small ellipsoidal scatterers. Radio Science, 21, 771-786.

Ulaby F.T., Moore R.K. and Fung A.K. (1982) Microwave Remote Sensing - Fundamentals and Radiometry. Volume I, Artech House: London, p. 456.

Wegmüller U. and Mätzler C. (1999) Rough bare soil reflectivity model. IEEE Transactions on Geoscience and Remote Sensing, 37, 1391-1395.

Wilheit T.T. (1978) Radiative transfer in a plane stratified dielectric. IEEE Transactions on Geoscience Electronics, 16, $138-143$. 
